\title{
Aromaterapia segura: Evitando a toxicidade no trabalho de parto
}

\author{
Safe aromatherapy: Avoiding toxicity in childbirth work \\ Aromaterapia segura: Evitar la toxicidade en el trabajo del parto
}

Rosana Serejo dos Santos

ORCID: https://orcid.org/0000-0003-3084-7539

Universidade Estácio de Sá, Brasil

E-mail: enfer.rosanaserejo@gmail.com

Solange Cristina Ferreira de Queiroz

ORCID: https://orcid.org/0000-0002-1716-0110

Universidade Federal do Piauí, Brasil

E-mail: solanghecf@gmail.com

Wendel Marcos Alves

ORCID: https://orcid.org/0000-0003-2194-7482

Universidade Estácio de Sá, Brasil

E-mail: marcosabreu78@hotmail.com

Letície Batista Lira

ORCID: https://orcid.org/0000-0003-4602-5177

Universidade Federal do Piauí, Brasil

E-mail: leticiebl@gmail.com

Edanielle da Silva Pereira Oliveira Felix

ORCID https://orcid.org/0000-0003-0761-5297

Prefeitura Municipal de Fortaleza, Brasil

E-mail: edanielleacs@gmail.com

Francisco Rondinele da Silva Felix

ORCID https://orcid.org/0000-0003-0616-1393

Prefeitura Municipal de Fortaleza, Brasil

E-mail: frondinelefelix@gmail.com

Suênia Maria da Silva Lima

ORCID: https://orcid.org/0000-0001-9610-6181

Universidade Estácio de Sá, Brasil

E-mail: sueniathibem@hotmail.com

Makellyne da Costa Mendes

ORCID: https://orcid.org/0000-0002-0071-3284

Universidade Estácio de Sá, Brasil

E-mail: makellynecm@ hotmail.com

Taiane Nayara Ribeiro da Silva

ORCID: https://orcid.org/0000-0002-2843-1342

Universidade Estácio de Sá, Brasil

E-mail:taiane642@gmail.com

Raquel de Sousa Sobral

ORCID: https://orcid.org/0000-0002-5682-4514

Universidade Estácio de Sá, Brasil

E-mail: raquelsobral95@gmail.com

Jaysse Kelly Amorim da Silva

ORCID: https://orcid.org/0000-0001-7747-4560

Universidade Estácio de Sá, Brasil

E-mail: mailto:jayssekelly12@gmail.com

Holiana Ruth Silva Cruz de Carvalho

ORCID: https://orcid.org/0000-0002-9484-6087

Universidade Estácio de Sá, Brasil

E-mail:holianaruth@hotmail.com

\begin{abstract}
Resumo
Objetivo: investigar as evidencias cientificas sobre os cuidados na utilização da aromaterapia no trabalho de parto. Metodologia: trata-se de uma revisão integrativa, foi realizada entre dezembro e janeiro de 2021, na base de dados National Library of Medicine National Institutes of Health, e nas bibliotecas virtuais Scientific Electronic Library Online, e Bibioteca Virtual em Saúde. Resultados e Discussão: Mantiveram-se no estudo 9, pois foram julgados coerentes com a proposta da revisão. A questão de segurança na utilização desses óleos essenciais parece de fácil abordagem, no entanto, a discussão a respeito de sua toxicidade é bem mais complexa. Deve-se considerar que os óleos essenciais exercem seus efeitos não apenas físico, mas também efeitos com atuação direta no cérebro, trazendo consequências muitas vezes
\end{abstract}


irreversíveis, os óleos puros frequentemente apresentam toxicidade elevada, tanto que, dentro das recomendações de uso, encontram-se as pequenas dosagens. Conclusão: a adoção da aromaterapia e de outras PIC associada ao resgate das suas bases científicas, possui benefícios no trabalho de parto validados por diversas evidencias cientificas.

Palavras-chave: Aromaterapia; Trabalho de parto; Sala de parto.

\begin{abstract}
Objective: to investigate the scientific evidence on care in the use of aromatherapy in labor. Methodology: this is an integrative review, it was carried out between December and January 2021, in the database National Library of Medicine National Institutes of Health, and in the virtual libraries Scientific Electronic Library Online, and Virtual Health Library. Results and Discussion: They remained in study 9, as they were considered consistent with the proposal of the review. The issue of safety in the use of these essential oils seems to be easy to approach, however, the discussion regarding their toxicity is much more complex. It should be considered that essential oils exert their effects not only physical, but also effects with direct action on the brain, bringing consequences that are often irreversible, pure oils often have high toxicity, so much so that, within the recommendations for use, they are found small dosages. Conclusion: the adoption of aromatherapy and other PIC associated with the rescue of its scientific bases, has benefits in labor validated by several scientific evidences.
\end{abstract}

Keywords: Aromatherapy; Labor obstetric; Delivery rooms.

\title{
Resumen
}

Objetivo: investigar la evidencia científica sobre el cuidado en el uso de aromaterapia en el trabajo de parto. Metodología: se trata de una revisión integradora, se realizó entre diciembre y enero de 2021, en la base de datos Biblioteca Nacional de Medicina de los Institutos Nacionales de Salud, y en las bibliotecas virtuales Biblioteca Electrónica Científica en Línea, y Biblioteca Virtual en Salud. Resultados y Discusión: Ellos permanecieron en el estudio 9, ya que se consideraron compatibles con la propuesta de revisión. El tema de la seguridad en el uso de estos aceites esenciales parece fácil de abordar, sin embargo, la discusión sobre su toxicidad es mucho más compleja. Se debe considerar que los aceites esenciales ejercen sus efectos no solo físicos, sino también efectos con acción directa sobre el cerebro, trayendo consecuencias que muchas veces son irreversibles, los aceites puros suelen tener alta toxicidad, tanto es así que, dentro de las recomendaciones de uso, se encuentran pequeñas dosis. Conclusión: la adopción de aromaterapia y otros PIC asociados al rescate de sus bases científicas, tiene beneficios en el trabajo validados por diversas evidencias científicas.

Palabras clave: Aromaterapia; Trabajo de parto; Salas de parto.

\section{Introdução}

Na história da assistência ao parto, observa-se uma transição onde inicialmente eram realizados partos em domicílios por mulheres conhecidas popularmente como parteiras, passando mais tarde a ser atendido em ambientes hospitalares. Com a evolução do cuidado à mulher, o trabalho de parto e parto vem permeando mudanças culturais, sociais e políticas importantes ao longo dos séculos (Gregorio \& Padilha, 2012).

Durante esse processo,o avanço tecnologico, trouxe seus beneficios para a qualidade da assistencia obstetrica nacional que devem ser reconhencida, em contrapartida essa tecnologia incluía rotinas desnecessarias como o jejum, o isolamento da parturiente no pré-parto sem a presença de acompanhante, ausência de liberdade para deambular, a cesárea, que caracterizam um modelo de assistência que pode perturbar e inibir o desencadeamento natural e fisiológico do parto; tornou-se sinônimo de doença e de intervenção médica, transformando-se em uma experiência marcada pela dor e impotência da mulher (Almeida; Acosta \& Pinhal, 2015).

Neste cenário, a Organização Mundial de Saúde - OMS e o Ministério da Saúde vêm propondo mudanças na assistência, incluindo o resgate do parto normal por meio de várias estratégias que possiblite a crianção de vinculo e suporte emocional à mulher, identificar e avaliar a dor, notificar à equipe médica, quando necessário, e principalmente implementar métodos não farmacológicos de alívio da dor (Almeida; Acosta \& Pinhal, 2015).

No tocante à temática do alívio da dor da parturiente, o uso dos métodos não farmacológicos é proposto como uma opção para substituição de analgésicos durante o trabalho de parto e o parto. Nessa perspectiva, esses cuidados são incentivados a partir da recomendação da prática de algumas ações não farmacológicas, essas práticas têm a finalidade de tornar o parto o mais natural possível, diminuindo as intervenções, cesarianas desnecessárias e a administração de fármacos (Gnatta et al., 2016). 
Terapias alternativas e complementares de saúde são definidas como qualquer sistema de medicina, prática ou produto que não fazem parte dos cuidados médicos convencionais, aromaterapia é uma terapia complementar utilizada durante o trabalho de parto para o alivio da dor e relaxamento muscular. Aromaterapia é uma técnica milenar, a qual utiliza óleos essenciais extraídos de diversas partes de plantas aromáticas (Silva et al., 2020). Os óleos essenciais são extraído com um alto nível de concentração, e ao serem absorvidos por inalação ou por uso tópico na pele os óleos acabam por estimular os receptores sensitivos estimulando o sistema nervoso parassimpático aumentando capaz de promover relaxamento (Silva et al, 2019).

Um óleo essencial apresenta uma composição complexa, algumasvezes, de centenas de diferentes compostos químicos, onde eles apresentamação sinérgica ou complementar entre si, modalizando sua atividade.Óleo essencial é um termo que designa substâncias aromáticas, geralmente deodor agradável e intenso, na maioria em forma líquida, encontradas em diferentesórgãos vegetais, são solúveis em solventes polares, por exemplo óleos fixos ecom solubilidade limitada em água, por isso as águas aromáticas (hidrolatos)apresentam o aroma da essência (Neves, 2011).

Com oobjetivo de oferecer acesso seguro ao uso das plantas medicinais e fitoterápicos, considerando a biodiversidade brasileira, o Ministério da Saúde instituiu a "Política Nacional de Plantas Medicinais e Fitoterápicos" (PNPMF) por meio do Decreto $\mathrm{n}^{\circ}$ 5.813, de 22 de junho de 2006. A PNPMF, torna-se relevante dentro da Política Nacional de Práticas Integrativas e Complementares da Saúde,considera o Brasil o país de maior biodiversidade do planeta, associada a uma rica diversidade étnica e cultural que detém um valioso conhecimento tradicional associado ao uso de plantas medicinais. A política possui como um dos seus princípios norteadores o uso sustentável da biodiversidade, o fortalecimento da produção oriunda da agricultura familiar e o incentivo à pesquisa e à indústria farmacêuticanacional (Brasil, 2006).

A partir da premissa de que a utilização da aromaterapia como intervenção terapeutica no trabalho de parto tras beneficios, torna-se necessário investigar quais os cuidados necessarios para manter o uso dos oleos essenciais seguros.

\section{Metodologia}

Este estudo trata-se de uma revisão integrativa da literatura, a partir do desenvolvimento da questão norteadora do estudo: "Quais as evidências científicas em relação aos cuidados no uso de oleos essenciais no trabalho de parto para minimizar complicações?". Elaborada através da adaptação da estrategia PICO (Galvão \& Ricarte, 2019) onde o P é a população, paciente ou problema =parturiente, $\mathrm{C}$ de conceito = aromaterapia, e para o elemento $\mathrm{C}$ (contexto) foi considerado sala de parto; conforme descrita na questão de estudo..

A busca dos estudos foi realizada entre dezembro e janeiro de 2021, na base de dados National Library of Medicine National Institutes of Health (PubMed), e nas bibliotecas virtuais Scientific Electronic Library Online (Scielo), e Bibioteca Virtual em Saúde MS (BVS- MS).

Para a busca dos estudos nas bases de dados selecionadas foram os descritores controlados selecionados nos Descritores em Ciência da Saúde (DeCS) usados para as bases disponíveis na BVS e Medical Subject Headings (MeSH) para o PubMed, conforme descrito no Quadro 1. 
Quadro 1. Termos de busca utilizados no processo de investigação e seleção dos estudos primários. Teresina, PI, Brasil. 2021.

\begin{tabular}{|c|c|c|c|c|}
\hline DESCRIÇÃO & PICo & COMPONENTES & $\begin{array}{c}\text { DESCRITORES } \\
\text { DECS }\end{array}$ & $\begin{array}{c}\text { DESCRITORES } \\
\text { MeSH }\end{array}$ \\
\hline Participantes & $\mathrm{P}$ & Parturiente & Gestantes & Pregnant Women \\
\hline Conceito & $\mathrm{c}$ & Óleos Essenciais & $\begin{array}{c}\text { Aromaterapia } \\
\text { Óleos Voláteis }\end{array}$ & $\begin{array}{c}\text { Aromatherapy } \\
\text { Oils, Volatile }\end{array}$ \\
\hline Contexto & $\mathrm{c}$ & Sala de parto & $\begin{array}{l}\text { Salas de Parto } \\
\text { Centro Obstétrico }\end{array}$ & Delivery Rooms \\
\hline
\end{tabular}

DeCS = Descritores em Ciências da Saúde. MeSH: Medical Subject Headings. Fonte: Dados da Pesquisa.

As publicações foram selecionadas segundo os seguintes critérios de inclusão: artigos disponíveis na integra, nas bases supracitadas, sem delimitação temporal, em qualquer idioma. Foras excluídos teses e dissertações, artigos pagos, artigos de revisão integrativas ou narrativa, e publicações duplicadas. A busca de dados foi realizada por meio de estratégia de busca desenvolvida na construção deste estudo, conforme descrita no Quadro 2.

Quadro 2. Estratégia de busca dos artigos nas bases de dados, 2021.

\begin{tabular}{|c|l|}
\hline $\begin{array}{c}\text { BASE DE } \\
\text { DADOS }\end{array}$ & \multicolumn{1}{c|}{ ESTRATÉGIA DE BUSCA } \\
\hline BVS & $\begin{array}{l}\text { (gestantes) OR (parturiente) OR ("Pregnant Women") AND (aromaterapia) OR ("Óleos Essenciais") } \\
\text { OR ("Oils, Volatile") OR (aromatherapy) AND ("Salas de Parto") OR ("Centro Obstétrico") OR } \\
\text { ("Delivery Rooms") }\end{array}$ \\
\hline SCIELO & $\begin{array}{l}\text { (gestantes) OR (parturiente) OR ("Pregnant Women") AND (aromaterapia) OR ("Óleos Essenciais") } \\
\text { OR ("Oils, Volatile") OR ( aromatherapy) AND ("Salas de Parto") OR ("Centro Obstétrico") OR } \\
\text { ("Delivery Rooms") }\end{array}$ \\
\hline PUBMED & ("Pregnant Women") AND ("Oils, Volatile") OR (aromatherapy) AND ("Delivery Rooms") \\
\hline
\end{tabular}

Fonte: Dados da Pesquisa.

Inicialmente, identificaram-se 34 artigos nas bibliotecas e base de dados selecionadas neste estudo. Após aplicar os critérios de inclusão e exclusão, 26 artigos permaneceram elegíveis. Após leitura criteriosa dos títulos, objetivos e resumos, 16 passaram por leitura indeliberada de todo o corpo do estudo, visando identificar quais abordavam a temática pesquisada e que, desta forma, adequava-se ao objetivo deste estudo. Ao final desta etapa, mantiveram-se no estudo 9, pois foram julgados coerentes com a proposta da revisão.

Nesta revisão buscou-se seguir a recomendação Preferred Reporting Items for Systematic Reviews and Meta-Analysis (PRISMA) para busca e seleção dos artigos, conforme Figura 1. A recomendação PRISMA trata-se de um documento desenvolvido para aumentar a qualidade das revisões sistemáticas e metanálises de ensaios clínicos randomizados e dos estudos não randomizados (Liberati et al 2009). 
Figura 1. Fluxograma do caminho percorrido na identificação e seleção de artigos, conforme recomendação PRISMA. Teresina, PI, Brasil. 2021.
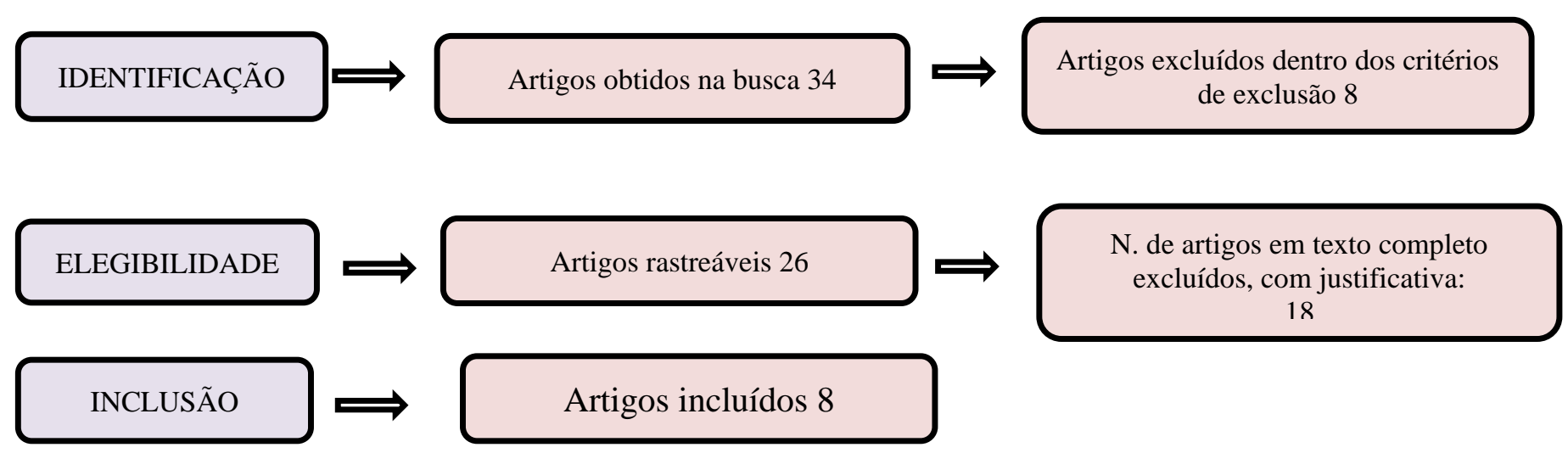

Fonte: Dados da Pesquisa.

\section{Resultados e Discussão}

Após o processo de identificação e inclusão, os estudos foram caracterizados os achados, segundo autores e ano de publicação, periódicos e idiomas, títulos, objetivos e principais achados, conforme disposto no Quadro 3.

Quadro 3. Caracterização dos artigos selecionados para análise de dados. Teresina, PI, Brasil, 2021.

\begin{tabular}{|c|c|c|c|c|}
\hline $\begin{array}{c}\text { Ano de } \\
\text { publicação }\end{array}$ & $\begin{array}{l}\text { Idiomas e } \\
\text { Periodicos }\end{array}$ & Títulos & Objetivos & Principais Achados \\
\hline 2013 & $\begin{array}{c}\text { Inglês } \\
\text { J Altern } \\
\text { Complement } \\
\text { Med } \\
\end{array}$ & $\begin{array}{l}\text { Physical and psychologic effects of } \\
\text { aromatherapy inhalation on } \\
\text { pregnant women: a randomized } \\
\text { controlled trial }\end{array}$ & $\begin{array}{l}\text { Esclarecer os efeitos físicos e } \\
\text { psicológicos da aromaterapia } \\
\text { inalatória em mulheres } \\
\text { grávidas }\end{array}$ & $\begin{array}{l}\text { A inalação de aromaterapia } \\
\text { com óleos essenciais foi } \\
\text { considerada eficaz para a } \\
\text { atividade do nervo } \\
\text { parassimpático }\end{array}$ \\
\hline 2014 & $\begin{array}{c}\text { Inglês } \\
\text { Women Birth }\end{array}$ & $\begin{array}{l}\text { The self-prescribed use of } \\
\text { aromatherapy oils by pregnant } \\
\text { women }\end{array}$ & $\begin{array}{l}\text { Realizar um estudo empírico } \\
\text { da prevalência e } \\
\text { características de mulheres } \\
\text { que usam óleos de } \\
\text { aromaterapia }\end{array}$ & $\begin{array}{l}\text { Há necessidade de maior } \\
\text { comunicação entre médicos e } \\
\text { pacientes em relação ao uso } \\
\text { de óleos de aromaterapia } \\
\text { durante a gravidez } \\
\end{array}$ \\
\hline 2014 & $\begin{array}{c}\text { Inglês } \\
\text { Iran Red } \\
\text { Crescent Med J }\end{array}$ & $\begin{array}{l}\text { The effect of lemon inhalation } \\
\text { aromatherapy on nausea and } \\
\text { vomiting of pregnancy: a double- } \\
\text { blinded, randomized, controlled } \\
\text { clinical trial }\end{array}$ & $\begin{array}{l}\text { Determinar o efeito da } \\
\text { aromaterapia por inalação de } \\
\text { limão nas náuseas e vômitos } \\
\text { durante a gravidez }\end{array}$ & $\begin{array}{l}\text { O aroma de limão pode ser } \\
\text { eficaz na redução de náuseas } \\
\text { e vômitos durante a gravidez }\end{array}$ \\
\hline 2014 & $\begin{array}{l}\text { Inglês } \\
\text { BJOG }\end{array}$ & $\begin{array}{l}\text { Healthcare professional views and } \\
\text { experiences of complementary and } \\
\text { alternative therapies in obstetric } \\
\text { practice in North East Scotland: a } \\
\text { prospective questionnaire survey }\end{array}$ & $\begin{array}{l}\text { Investigar o uso de terapia de } \\
\text { medicamentos } \\
\text { complementares e } \\
\text { alternativos (CAMs) por } \\
\text { profissionais de saúde do } \\
\text { Reino Unido envolvidos no } \\
\text { cuidado de mulheres grávidas }\end{array}$ & $\begin{array}{l}\text { identificou que aqueles que } \\
\text { recomendaram CAMs eram } \\
\text { significativamente mais } \\
\text { propensos a serem parteiras } \\
\text { que estavam no cargo por } \\
\text { mais de } 5 \text { anos, receberam } \\
\text { treinamento. }\end{array}$ \\
\hline 2016 & $\begin{array}{c}\text { Inglês } \\
\text { Complement } \\
\text { Ther Clin Pract }\end{array}$ & $\begin{array}{l}\text { The effect of aromatherapy with } \\
\text { lavender essence on severity of } \\
\text { labor pain and duration of labor in } \\
\text { primiparous women. }\end{array}$ & $\begin{array}{l}\text { Investigar o efeito da } \\
\text { inalação da essência de } \\
\text { lavanda na gravidade da dor } \\
\text { do parto e na duração do } \\
\text { trabalho de parto. } \\
\end{array}$ & $\begin{array}{l}\text { Os resultados mostraram que } \\
\text { a diferença na dor do parto } \\
\text { antes e depois da intervenção } \\
\text { em dois grupos foi } \\
\text { significativa. }\end{array}$ \\
\hline 2017 & $\begin{array}{l}\text { Inglês } \\
\text { J Altern } \\
\text { Complement } \\
\text { Med }\end{array}$ & $\begin{array}{l}\text { Effects of Aromatherapy Massage } \\
\text { on Pregnant Women's Stress and } \\
\text { Immune Function: A Longitudinal, } \\
\text { Prospective, Randomized } \\
\text { Controlled Trial }\end{array}$ & $\begin{array}{l}\text { Examinar os efeitos da } \\
\text { massagem com aromaterapia } \\
\text { no estresse e na função } \\
\text { imunológica das mulheres } \\
\text { durante a gravidez. }\end{array}$ & $\begin{array}{l}\text { Apresentou evidências de que } \\
\text { a massagem com } \\
\text { aromaterapia pode diminuir } \\
\text { significativamente o estresse } \\
\text { e melhorar a função } \\
\text { imunológica em mulheres } \\
\text { grávidas. }\end{array}$ \\
\hline
\end{tabular}




\begin{tabular}{|l|c|l|l|l|}
\hline 2017 & Inglês & $\begin{array}{l}\text { Changes in salivary oxytocin after } \\
\text { inhalation of clary sage essential oil } \\
\text { scent in term-pregnant women: a } \\
\text { feasibility pilot study. }\end{array}$ & $\begin{array}{l}\text { Avaliar a viabilidade, } \\
\text { eficácia limitada, praticidade } \\
\text { e aceitabilidade) de nosso } \\
\text { protocolo de intervenção } \\
\text { envolvendo inalação do } \\
\text { cheiro de óleo essencial }\end{array}$ & $\begin{array}{l}\text { O protocolo de intervenção } \\
\text { apresentou boa aceitabilidade. } \\
\text { Em termos de praticidade, ele } \\
\text { pode ser ajustado para } \\
\text { permitir a coleta de uma } \\
\text { quantidade suficiente de } \\
\text { saliva para a medição ideal do } \\
\text { nível de oxitocina. }\end{array}$ \\
\hline $\mathbf{2 0 1 9}$ & Português & $\begin{array}{l}\text { Evidências científicas sobre } \\
\text { métodos não farmacológicos para } \\
\text { alívio da dor no parto } \\
\text { Enferm. } \\
\text { (Online) }\end{array}$ & $\begin{array}{l}\text { Identificar na literatura } \\
\text { estudos sobre a eficácia de } \\
\text { métodos não farmacológicos } \\
\text { na redução da dor do parto }\end{array}$ & $\begin{array}{l}\text { As terapias térmicas } \\
\text { contribuem para a analgesia } \\
\text { local de regiões afetadas pela } \\
\text { dor }\end{array}$ \\
\hline $\mathbf{2 0 2 0}$ & $\begin{array}{l}\text { Ingles } \\
\text { J Obstet }\end{array}$ & $\begin{array}{l}\text { Aromatherapy intervention on } \\
\text { anxiety and pain during first stage } \\
\text { labour in nulliparous women: a } \\
\text { systematic review and meta- } \\
\text { analysis. }\end{array}$ & $\begin{array}{l}\text { Avaliar e resumir as } \\
\text { evidências disponíveis } \\
\text { derivadas de ensaios clínicos } \\
\text { randomizados sobre os } \\
\text { efeitos da aromaterapia na } \\
\text { dor do parto e no alívio da } \\
\text { ansiedade. }\end{array}$ & $\begin{array}{l}\text { Identificaram evidências mais } \\
\text { confiáveis que validam que a } \\
\text { aromaterapia pode diminuir } \\
\text { significativamente a dor do } \\
\text { parto nas fases ativa inicial e } \\
\text { ativa tardia }\end{array}$ \\
\hline
\end{tabular}

Fonte: Dados da Pesquisa.

Pela análise dos artigos selecionados, na Biblioteca Virtual em Saúde (BVS) todos os artigos selecionados podem ser encontrados, embora exista a proporção de duplicação em outras bases de dados, com maior concentração e maior frequência nos últimos cinco anos, uma vez que a partir de 2010 encontramos mais artigos e com menor intervalo de tempo de publicação entre um e outro, com predomínio no ano de 2014 e 2017 (representando 33,33\% e 22,22\% respectivamente).

No presente estudo, o foco foi no aspecto físico e efeitos psicológicos da aromaterapia em mulheres em trabalho de parto no uso de terapia complementar, aromaterapia, quais os cuidados para que não ocorra complicações. Compreender os cuidados para evitar a toxicidade, analisando o risco benefício no uso de óleos essenciais.

Para Castro e Figueiredo(2019) a aromaterapia é uma prática popular utilizada no Brasil desde antes da colonização foi institucionalizada, tendo o seu uso popular comprovado através de métodos da ciência moderna e após a sua comprovação passaram a ser interessantes para a indústria farmacêutica. Por outro lado a inclusão das plantas medicinais no SUS e a criação da Política Nacional de Plantas Medicinais e Fitoterápicos têm potencial para gerar transformações no campo da saúde, por ser mais uma opção de tratamento que carrega um significado de cura, prevenção e tratamento diferente das práticas médicas convencionais, cada vez mais baseadas no uso intensivo de tecnologias nas suas práticas.

A questão de segurança na utilização desses óleos essenciais parece de fácil abordagem, no entanto, a discussão a respeito de sua toxicidade é bem mais complexa. Deve-se considerar que os óleos essenciais exercem seus efeitos não apenas físico, mas também efeitos com atuação direta no cérebro, trazendo consequências muitas vezes irreversíveis, os óleos puros frequentemente apresentam toxicidade elevada, tanto que, dentro das recomendações de uso, encontram-se as pequenas dosagens.

Deve-se ressaltar que a inalação prolongada de OE pode causar dores de cabeça, náuseas, alergias e irritação na pele, por isso priorizar sempre o respeito ao limite de cada mulher é algo importante. Acrescenta-se que a utilização dessas terapias complementares pela Enfermagem é fundamental para estabelecer a autonomia profissional, pois o conhecimento e divulgação destes métodos são a base para dinamizar o seu caráter científico e contribuir para o planejamento de pesquisas clínicas.

Em seus estudos Souza et al., (2020) destacam que o modelo de saúde continua centrado na doença. No entanto, dentre as Práticas Integrativas e Complementares (PICS) destacam-se a musicoterapia, a hidroterapia, a aromaterapia, reflexologia podálica, escalda-pés, uso de plantas medicinais, foram empregadas para o alívio de sintomas físicos, dores em geral, com benefícios emocionais e psicológicos, no processo de parturição, durante o pré-natal, neoplasias e nos sinais de climatério. Os profissionais da saúde que frequentemente utilizam essas práticas no cuidado à saúde da mulher são médicos, enfermeiros, 
fisioterapeutas e doulas.

Ainda de acordo com Souza et al., (2020) Apesar de não fazer parte dos métodos farmacológicos, as PICS provocam na mulher que a utiliza frequentemente uma mudança de humor ou comportamento, impactando dessa forma nas atividades de vida diárias. Dessa forma, é bom pensar que para a aplicação dessas atividades terapêuticas são necessários profissionais nos serviços de saúde, que sejam qualificados e especializados e, a partir disso, trazer a perspectiva deles quanto a utilização das terapias complementares.

Revela-se tamanha importância da utilização de métodos não farmacológicos, como é o caso da aromaterapia, para alívio da dor, ansiedade, estresse, dentre outros sintomas desconfortáveis inerentes ao trabalho de parto, podendo a aromaterapia ser aplicada de forma única ou associada a outras terapias como a massagem, considerando a individualidade de cada parturiente e para tal a seleção dos óleos essenciais deve ser criteriosa, bem como sua via de aplicação.

O manejo das PICs, em especial a aromaterapia, trazem ao contexto hospitalar tecnologias não-farmacológicas, que elevam a assistência à parturiente ao âmbito da naturalidade e protagonismo da mulher, favorecendo seu empoderamento. faz-se necessário a difusão dos benefícios da utilização da aromaterapia no contexto obstétrico, assim como incentivos à educação permanente e estímulos a produção científica para ampliação da implementação de métodos não-medicalizados por parte dos profissionais de saúde, para que no futuro mais instituições de saúde ofereçam tais estratégias na prestação do cuidado, valorizando autonomia, exibindo seus benefícios e garantindo uma terapêutica focada nas necessidades de saúde singulares de cada mulher (Paganini, 2013).

Em seus estudos Igarashi (2013) buscou esclarecer os efeitos físicos e psicológicos da aromaterapia inalatória em mulheres grávidas. Foram selecionados e dentre estes óleos essenciais com alto teor de linalol e acetato de linalila que podem ser utilizados durante a gestação, foi utilizado o preferido pela participante. O estudo foi realizado em um departamento ambulatorial de ginecologia em um hospital em Kyoto, Japão.

A inalação de aromaterapia com óleos essenciais contendo acetato de linalila e linalol mostrou-se eficaz para a atividade do nervo parassimpático, com base em uma comparação intragrupo. No entanto, com base na comparação entre os grupos, nenhuma diferença substancial foi observada; portanto, mais estudos são necessários no futuro (Igarashi, 2013).

Os cuidados no uso dos óleos essenciais, é importante certificar-se quanto à aquisição de óleo essencial puro, 100\% natural vegetal, para não levar, por engano, a essência sintética. Ela não oferece os mesmos benefícios do óleo e pode causar efeitos deletérios à saúde, o produto tem que vir conservado em frascos de cor escura (âmbar), azul ou verde, para que sejam protegidos da ação da luz UV, pois podem sofrer perdas se expostos a esses raios (Nascimento \& Prade, 2020)

De acordo com Nascimento e Prade (2020), algumas recomendações de segurança são necessárias, como: Não é indicado o uso oral dos óleos essenciais; A prática acompanhada por profissional capacitado é sempre recomendada, principalmente para o uso dos óleos essenciais em crianças, idosos, grávidas e pessoas com doenças crônicas, sempre comunicar ao profissional de saúde que está fazendo uso da aromaterapia, pois alguns óleos essenciais podem promover interação medicamentosa e ou efeitos adversos se não utilizados adequadamente. Pessoas reconhecidamente hipersensíveis a óleos essenciais devem evitá-los. Ocorrendo reação de hipersensibilidade, o uso deve ser suspenso.

$\mathrm{Na}$ gravidez, a necessidade de seguir as diretrizes de segurança para uso de óleos essenciais. Pois os componentes químicos presentes nos aromáticos cruzam a barreira placentária e podem atingir o bebê. Cabe salientar, que se o óleo essencial for diluído, a quantidade de componentes que irá atravessara pele da gestante e atingir na placenta é mínima.

As gestantes possuem uma ativação maior da melanina, o que pode ocasionar queimaduras quando expostas ao sol forte, estão contraindicados a aplicação de óleos aromáticos que foram extraídos por prensagem, porque estes contêm uma quantidade maior de furanocumarinas. Sempre é importante observar que durante a gravidez a mulher pode reações alérgicas que até o momento não havia apresentado, sendo, portanto, necessário fazer um teste, aplicando-se o óleo aromático na parte interna do antebraço, pois ali a pele é mais fina. Após a aplicação observar por no mínimo 10 minutos, se após este período não apresentar nenhuma reação o 
óleo poderá ser utilizado na gestante.

Em seus estudos Liao et al., (2020) resumem evidências derivadas de ensaios clínicos randomizados em que a aromaterapia foi realizada como um método analgésico de suporte durante o parto. Os resultados identificaram evidências mais confiáveis que validam que a aromaterapia pode diminuir significativamente a dor do parto nas fases ativa inicial e ativa tardia.

\section{Conclusão}

Percebe-se um vasto campo propício para utilização da aromaterapia, uma vez que é de baixo custo, porém a implantação desta prática por parte da Enfermagem ainda é pouco difundida Faz-se necessário a ampliação dos estudos a cerca desta temática, bem como disseminar informações sobre sua eficácia e benefícios para com os profissionais que prestam assistência à parturiente, incentivar a adoção de métodos não farmacológicos nas práticas clínicas por parte dos profissionais da saúde, assim como as instituições que oferecem.

\section{Referências}

Almeida, J. M. D., Acosta, L. G., \& Pinhal, M. G. (2015). Conhecimento das puérperas com relação aos métodos não farmacológicos de alívio da dor do parto. Revista Mineira de Enfermagem, 19(3), 711-724.

Brasil. Ministerio da Saúde (2006). Política Nacional dePlantas Medicinais eFitoterápicos. Brasília. <http://bvsms.saude.gov.br/bvs/publicacoes/politica_nacional_fitoterapicos.pdf >.

Castro, M. R., \& Figueiredo, F. F. (2019). Saberes tradicionais, biodiversidade, práticas integrativas e complementares: o uso de plantas medicinais no sus. Hygeia - Revista Brasileira De Geografia Médica E Da Saúde, 15(31), 56 - 70. https://doi.org/10.14393/Hygeia153146605.

Gnatta, J. R., Kurebayashi, L. F. S., Turrini, R. N. T., \& Silva, M. J. P. D. (2016). Aromaterapia e enfermagem: concepção hi stórico-teórica. Revista da Escola de Enfermagem da USP, 50(1), 127-133.

Gregório, V. R. P., \& Padilha, M. I. C. D. S. (2012). History of care to women in the Maternity Carmela Dutra, Florianópolis-SC, Brazil (1956-2001). Revista brasileira de enfermagem, 65(5), 767-774.

Igarashi, T. (2013). Physical and psychologic effects of aromatherapy inhalation on pregnant women: a randomized controlled trial. The Journal of Alternative and Complementary Medicine, 19(10), 805-810.

König, S. (2020). Óleos essenciais: eventos adversos e segurança na indicação. http://hdl.handle.net/11624/3068.

Liao, C. C., Lan, S. H., Yen, Y. Y., Hsieh, Y. P., \& Lan, S. J. (2020). Aromatherapy intervention on anxiety and pain during first stage labour in nulliparous women: a systematic review and meta-analysis. Journal of Obstetrics and Gynaecology, 1-11.

Mascarenhas, V. H. A., Lima, T. R., Silva, F. M. D., Negreiros, F. D. S., Santos, J. D. M., Moura, M. Á. P., \& Jorge, H. M. F. (2019). Evidências científicas sobre métodos não farmacológicos para alívio a dor do parto. Acta Paulista de Enfermagem, 32(3), 350-357.

Nascimento, A., \& Prade, A. C. K. (2020). Aromaterapia: o poder das plantas e dos óleos essenciais. Fiocruz-PE.

Neves, J. S. (2011) Aromaterapia: um tema para o ensino de química. 28 f., il. Monografia. Universidade de Brasília, Brasília.

Paganini, T. (2013). Bem-estar e qualidade de vida: a aromaterapia no cuidado ao estresse.

Safajou, F., Shahnazi, M., \& Nazemiyeh, H. (2014). The effect of lemon inhalation aromatherapy on nausea and vomiting of pregnancy: a double-blinded, randomized, controlled clinical trial. Iranian Red Crescent Medical Journal, 16(3).

Sibbritt, D. W., Catling, C. J., Adams, J., Shaw, A. J., \& Homer, C. S. (2014). The self-prescribed use of aromatherapy oils by pregnant women. Women and birth, 27(1), 41-45.

Silva, M. A. N., Coelho, O. P., Neves, P. R., de Souza, A. R. L., da Silva, G. B., \& Lamarca, E. V. (2020). Acerca de pesquisas em aromaterapia: usos e benefícios à saúde. Revista da Universidade Ibirapuera Jan/jun, (19), 32-40.

Silva, M. A.,Sombra, I. V.S, Jacinto da Silva, J. S., Bernardino da Silva, J. C., de Melo Dias, L. R. F., Ferreira Calado, R. S., \& de Menezes Silva, G. A. (2019). Aromaterapia para alívio da dor durante o trabalho de parto. Journal of Nursing UFPE/Revista de Enfermagem UFPE, 13(2).

Souza, V. A. de, Machado, G. N., Arrué, A. M., Luzardo, A. R., Jantsch, L. B., \& Danski, M. T. R. (2020). Integrative and Complementary Practices in women's health care. Research, Society and Development, 9(8), e81985379.

Stewart, D., Pallivalappila, A. R., Shetty, A., Pande, B., \& McLay, J. S. (2014). Healthcare professional views and experiences of complementary and alternative therapies in obstetric practice in $\mathrm{N}$ orth $\mathrm{E}$ ast $\mathrm{S}$ cotland: a prospective questionnaire survey. BJOG: An International Journal of Obstetrics \& Gynaecology, 121(8), 1015-1019. 
Research, Society and Development, v. 10, n.6, e27210615827, 2021

(CC BY 4.0) | ISSN 2525-3409 | DOI: http://dx.doi.org/10.33448/rsd-v10i6.15827

Tadokoro, Y., Horiuchi, S., Takahata, K., Shuo, T., Sawano, E., \& Shinohara, K. (2017). Changes in salivary oxytocin after inhalation of clary sage essential oil scent in term-pregnant women: a feasibility pilot study. BMC research notes, 10(1), 1-7.

Yazdkhasti, M., \& Pirak, A. (2016). The effect of aromatherapy with lavender essence on severity of labor pain and duration of labor in primiparous women. Complementary therapies in clinical practice, 25, 81-86. 